\title{
A Review Paper on Automatic Meter Reading and Instant Billing
}

\begin{abstract}
Bhushan D. Sawarkar ${ }^{1}$, Mrs. Snehal S. Golait ${ }^{2}$
M.E. Student, Department of Computer Technology, Priyadarshini College of Engineering, Nagpur (MH), India ${ }^{1}$ Assistant Professor, Department of Computer Technology, Priyadarshini College of Engineering, Nagpur (MH), India ${ }^{2}$

Abstract: The existing systems are either an electronic energy meter or an electro-mechanical meter which are currently in use is limited to record up to $\mathrm{kWh}$ units. The $\mathrm{kWh}$ units recorded by meter readers monthly, on foot which need to be processed by a meter reading company. For processing the meter reading, company needs to link each recorded usage data to the particular account holder and then determine the amount owed by means of the specific tariff in use. On basis of various platforms researchers proposed many system for Automatic Meter Reading (AMR). There are various wire-based AMR systems like Power Line Carrier (PLC) and Telephone Line Network (optical/ cable) and wireless AMR systems such as E-metering systems based on GPRS, Bluetooth, GSM. Design of an Electric Energy Meter for long-distance data information transfers which based upon GPRS, but this system can't be implemented so easily because the regular use of GPRS is still a dream to the common man. A GSM based Energy meter with instant billing facility is introduced is efficient, but still the problem of missing SMS will degrade the accuracy and performance. A more reliable and user friendly system by creating web portal for multiple access using the advanced Visual studio .net frame work which will manage the data efficiently even if there is loss of SMS. It makes the design different from the previous proposals and also increases the throughput. The GSM/GPRS channel is a very useful means of communication as sending data as SMS turns out to be a very handy tool, due to its good area coverage capability and cost effectiveness. The front end web portal is User friendly and any employee with minimum knowledge of computers can work on this software. Employees can read the meter by sitting in their office.
\end{abstract}

Keywords: Automatic Meter Reading System (AMRS), GSM, PIC, Short Messaging System (SMS), Visual Studio .NET

\section{INTRODUCTION}

We never think the life without the electrical power switches on/off, which reduces required time and increases because human survival and progress totally depends over throughput.

it. The onset of electrification provides opportunities for This paper presents an implementation methodology for a new and more efficient metering technologies to be wireless automatic meter reading system (WAMRS) implemented and the future residential development needs. incorporating the widely used GSM/ GPRS network. In Previously used Power Line Communication [PLC] for data communication has many limitations like complexity of network, cost of installation and maintenance. For different type of services we require different frequency bands and power line uses $50 \mathrm{~Hz}$ also unable to support higher frequency bands. The power line is uncovered since losses also increases on the other hand interference between different channels is also big problem. There are some general problems like highly person dependent, human errors can't be avoided, accessibility in rural zone, billing on monthly basis and its processing takes excess time. The Digital Telewattmeter System is an example of microprocessor based meter. The meter was designed to transmit data on monthly basis to a remote central office through a dedicated telephone line and a pair of modems. Next was to use a DSP based meter to measure the electricity consumption of the customers in the residential area. So there is increased demand for Wireless Automatic Meter Reading (WAMR) systems which automatically collecting consumption, diagnostic, and status data from metering devices and transferring that data to a central database for billing, troubleshooting, and processing. It mainly reduces the human efforts as well as manual errors, provides real time correct consumption, Remote power many countries GSM and GPRS network is widely known for its vast coverage area, cost effectiveness and also for its competitive ever growing market. The system includes a microcontroller, which transmits the power consumption values periodically, via an existing GSM/GPRS network, to a master station. To maintain transparency between consumer and company we suggest a method where we utilize telecommunication systems for automated transmission of data to facilitate bill generation at the server end and also to the customer via SMS, Email. A new interactive, user friendly graphical user interface is developed using Microsoft visual studio .NET framework. With proper authentication, users can access the developed web page details from anywhere in the world.

In this paper, following a brief introduction of latest Automatic Meter Reading Systems described and summarized. The existing problems and future research directions are also discussed.

\section{RELATED WORKS AND METHODOLOGIES USED}

\section{History Of Meters}

With improvement of national power and country's economy, demands and requirements also 
increases of various entities like power and proper overcoming the "silent node" problem in AMR systems. management is also necessary for that the conventional Also, a two-state transition Markov model is used to energy metering systems are available. In last few years, simulate a meter unit with such a "silent node" problem in metering devices have gone through much improvement, a PLC network. The processing time model is established and are expected to become even more sophisticated, on a meter topology of three-level clustering. By using offering more and more services. Past year meters, like these models for our computer simulations, the electromechanical devices with poor accuracy and lack of performance of our proposed schemes can be evaluated in configurability with some challenges of theft detection, terms of reliability and efficiency. providing limited amount of energy consumption on site.
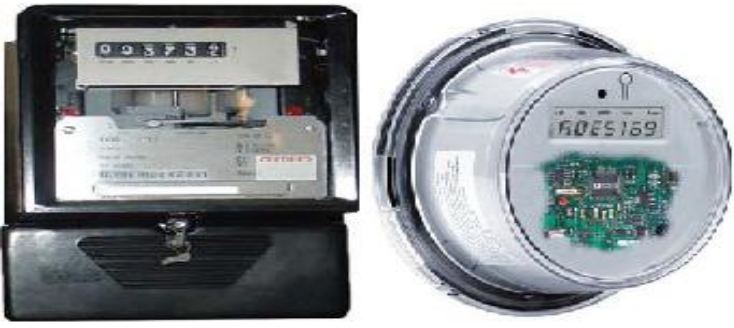

Fig.1 Traditional Electromechanical meter Vs Electronic meter.

Recent developments in this direction seem to provide energy efficient metering technologies that are more precise and accurate, error free, etc. The implementation of WAMRS provides with many vital features as compared with the analog utility meter reading with man power. Some of these features are listed below,

Higher speed.

Improved load profile.

Automatic billing invoice.

Real time energy cost.

Load management.

Alarm warning.

Remote power switches on/off.

Tamper detection.

Bundling with water and gas.

WAMRS provides full duplex communication between the electricity company and the load by sending in a lot of power parameters and control signal to reach the goal of load management and power demand control also maintains the transparency by sending monthly consumption to user by SMS also user can check his usages from any place on any time through web portal made by any frameworks.

\section{Methodologies}

Q. Gao, J. Y. Yu, P. H. J. Chong proposed two algorithms, namely clustered simple polling and neighbor relay polling, for solving the typical "silent node" problem in automatic meter reading (AMR) systems using powerline communications (PLC)-access networks. A "silent node"[1] is a meter unit that cannot receive signals directly from the BS or that cannot send its metering data back directly to the BS. Here the factors responsible for an unreliable rate of data collection and significant time delay are the significant level of signal attenuation along cables, the noisy power-line environment, and the changing inhome impedance. These schemes are tested through computer simulations to be both effective and efficient in
Polling is normally implemented in a system with a central controller that is called a base station (BS). The BS sends a "polling message" to each network station, in accordance with a round-robin procedure or any other cyclic order. When a network station receives a polling message from the BS and it has data to send back to the BS, it can transmit its data for a predefined time period. Then, the network station also transmits an acknowledgement $(\mathrm{ACK})$ at the end of a packet transmission to inform the BS that its data transmission is completed before the stipulated time limit. If the network station has no data to send, it just sends an ACK to the BS to inform that it has no data to send. Afterwards, the BS polls the next network station in the same cycle immediately. If the BS cannot hear anything from the polled station after a stipulated time period, it will poll the next station anyway. Hence, the next network station in the same cycle can be polled. The NRP is a more effective mechanism because it can reach a nearly $100 \%$ data-collection success rate under normal network conditions. In some cases, the NRP cannot guarantee the data-collection time well below 30 min due to additional time for the repolls. Under some extremely adverse network conditions, the meter units belonging to the same building and the same phase of the same transformer cannot be guaranteed as neighbors because they may not be able to communicate directly with each other. Hence, the polling message and metering data may need to go through more than one relaying meter.

Mpendulo Ndlovu, Ling Cheng Proposed Power-line communication offers a networking communication over existing power lines and finds important applications in smart grid, home \& business automation and automatic meter reading. However, the power-line channel is one of the harshest known communication channels currently in use and it requires robust forward error correction techniques. Powerful decoding algorithms tend to be complex and increase latency while robust modulation schemes offer lower data rates. The presented work extends the existing narrow-band power-line communication forward error correction scheme of concatenated Reed-Solomon Convolution codes in OFDM framework by introducing permutation codes as an inter subcarrier encoding scheme in OFDM to combat narrowband interference and carrier frequency offsets.

Permutation encoded OFDM has been presented as an alternative modulation scheme for narrow-band powerline communication. While it uses more bandwidth than OFDM, it performs much better in the presence of carrier frequency offsets. OFDM-MFSK[6] is a similar scheme that was proposed by other researchers as another alternative. Although this scheme's performance is similar 
to that of the presented scheme, it is less efficient in terms of bandwidth utilization. Permutation encoded OFDM uses up to 103 subcarriers in a 256 length IFFT window, giving $40 \%$ bandwidth efficiency. On the other hand, OFDM-MFSK uses a maximum of only 64 subcarriers, translating to an efficiency of $25 \%$. Permutation encoded OFDM [6] therefore gives an improvement of $60 \%$ in terms of bandwidth efficiency on this scheme. In addition, MFSK-based schemes are limited to sending only $\log 2 \mathrm{M}$ bits for every $M$ frequency, while PE OFDM can employ higher order M-PSK schemes in OFDM subcarriers and achieve higher throughputs.

Mohamed Chaker Bali, Chiheb Rebai praposed the design of advanced physical layer for narrowband power line communications (NB-PLC) based on coded modulation[7]. The coding modulation scheme is described and decoding process based on soft decoding is proposed. Using this innovative scheme, the decoder performs a gain of $3 \mathrm{~dB}$ at symbol error rate (SER) equal to 10-3 over traditional receiver with hard decoding. In the implementation analysis, both the digital signal processor (DSP) resources usage and the maximum data rate are considered. It is concluded that a transmission rate of 2.4 kbps is reached for a robust NB-PLC and available resources can be used for full protocol stack implementation. This architecture greatly simplifies both the hardware and software design implementation of overall coding modulation NB-PLC modem.

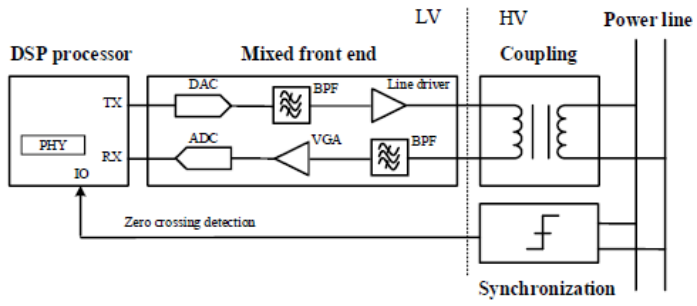

Fig 2. NB-PLC modem functional block diagram

A new decoding process for coding modulation is proposed and analyzed under frequency selective channel through numerical simulations. The proposed soft decoder achieves $3 \mathrm{~dB}$ of gain for SER equal to 10-3 compared to hard decoder. Finally, a practical and optimized DSP implementation of NB-PLC modem [7] is presented. This DSP-based modem offers to users' flexibility and programmability to design full AMR solution.

K. S. K. Weranga, D. P. Chandima focuses on smart metering for next generation energy efficiency and conservation. The discussion here is based on implementation of new methodologies to achieve energy efficiency via two way interaction. Improved measurement technology by displaying all per phase information and three phase information on LCD at the meter side, automatic meter reading, power quality and exported energy measuring capability, energy and cost forecasting for better energy conservation are the key methodologies of this research. The digital meter was developed using ADE7758 energy metering chip, 18F452 PIC microcontroller and PCF8583 real time clock IC. The data are sent to a remote server via SMS using SIM900 GSM module. The server handles the incoming SMS, processes, Copyright to IJARCCE displays and stores the required information. Energy consumption and its cost, average daily energy consumption and cost prediction for the month are calculated in the server side.

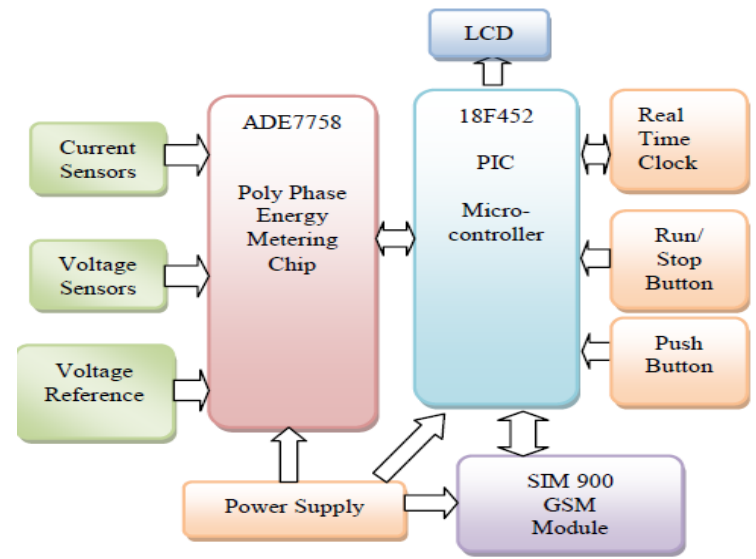

Fig. 3. Block diagram of the Smart meter

Smart meters [4] are a better option for energy efficiency in today's world. They are just the start of the merging of information technology, wireless networking of the home electrical appliances and the efficient use of electrical power, designed to save costs and the environment. There are still many challenges on smart metering. Some of them are technology, standardization, demand management approach and data volumes. The customer relationship with smart meters may be affected in the long-term if there are no strong initiatives. If consumers do not use the data from smart meters to amend their energy consumption over the long-term, smart meter projects will ultimately fail. In countries where they don't have a good load profile, smart metering will be a good alternative to achieve energy efficiency. That will help to cut down those peaks to some extents through demand side management and to lower the generation cost. Power distributers will also benefit as the information provided by smart meters. It will help them to monitor energy across their networks and manage load more effectively. For an example he can monitor the voltage variations, outages, power factors for each consumer and decide the quality of his supply. On the other hand this data can points the location of outages. Therefore the operator can easily schedule the maintenance crew to take necessary actions. Faster respond for the small outages will help to provide a good customer service. Meanwhile the real time consumption data and aggregation of consumer profiles allows for a more precise forecast of energy consumption which improves the network management and planning processes.

Nhat-Quang Nhan, Minh-Thanh Vo, Tuan-Duc Nguyen, Huu-Tue Huynh proposed paper over Automatic Meter Reading (AMR) [3] systems have been studied and largely exploited in many developed countries, using different communication techniques such as PLC, Wi-Fi, Telephone Line, etc. However, recent researches have shown that collecting data using Wireless Sensor Network (WSN) is the future technology for AMR system. In fact, researchers have implemented the technique gathering data using WSN. Nevertheless, this kind of network has different 
characteristics compared with the traditional WSN. This work proposes the metering network topology corresponding to the household distribution in most of developing countries. Then, it presents the data collection method for this topology and points out the essential of Mobile Data Collector (MDC) for this network. Finally, it shows the effect of MDC speed on the reliability of electricity data collection using NS2 simulation. The simulation results indicate that the proposed data collection method has an improvement over the traditional MDC data collection using ZigBee wireless devices.

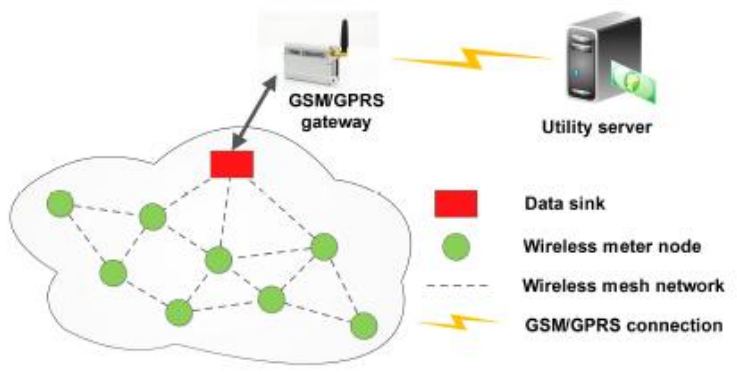

Fig.4. Data collection method for urban model

This paper explained the possibility of using AMR systems in developing countries. For this purpose, we have proposed WSN as a potential solution for this problem. To show that WSN is in fact a good solution, we have method determined a convenient network topology applied to the problem of electricity meter data collection. Finally, we have proposed a practical structure for efficient data collection in this network topology. The performance of the overall system is evaluated by simulations with different MDC speeds and different sensing techniques. The analysis of obtained results shows that the proposed method offers a significant improvement on delay of data transfer with high MDC speed, compared to the traditional use of ZigBee.

Subhashis Maitra praposed a new concept of energy meter was discussed before, where maximum demand of energy of a consumer will be indicated in the meter used by the consumer. After exceeding the maximum demand, the meter and hence the connection will automatically be disconnected by an embedded system inserted in the meter itself. According to the maximum demand, the consumer will purchase a cash-card [2] of amount depending on the consumption of energy and after the full consumption, the consumer again has to purchase another cash-card or recharge the same and thus the hassle related to go to the billing office, to stand in a long queue and to submit the bill, can be avoided. Also this system helps to eliminate the draw backs of billing management system, such as to take the reading from the meter, to create the bill, to print the bill, to send the bill to the proper address and to collect the amount for the bill. Hence this system can effectively reduce the man power required to a great extent. Also a new concept of a distributor has been dealt here which is used to disconnect a line if the energy consumption per day of a consumer greatly exceeds pre-demand energy consumption per day. With the help of this system electric supply authority can detect a power hacker also.
There is no one needs to go to the Electric supply office, to waste time by standing in a long queue and ultimately to pay the bill. At the same time it will be helpful to the supplier also. After implementing this system, the agent of the supply authority has not to go to the user end, not to prepare the bill and not to send the bill to the proper address. The proposed system increases customer convenience, decreases man power, reduce the risk of theft of cash, increases reliability, increases safety and security, reduces the cost of bill management system etc.

Sudhish N George proposed the technology of e-metering (Electronic Metering) has gone through rapid technological advancements and there is increased demand for a reliable and efficient Automatic Meter Reading (AMR) system. This paper presents the design of a simple low cost wireless GSM [5] energy meter and its associated web interface, for automating billing and managing the collected data globally. The proposed system replaces traditional meter reading methods and enables remote access of existing energy meter by the energy provider. Also they can monitor the meter readings regularly without the person visiting each house. A GSM based wireless communication module is integrated with electronic energy meter of each entity to have remote access over the usage of electricity. A PC with a GSM receiver at the other end, which contains the database acts as the billing point. Live meter reading from the GSM enabled energy meter is sent back to this billing point periodically and these details are updated in a central database. A new interactive, user friendly graphical user interface is developed using Microsoft visual studio .NET framework and C\#. With proper authentication, users can access the developed web page details from anywhere in the world. The complete monthly usage and due bill is messaged back to the customer after processing these data.

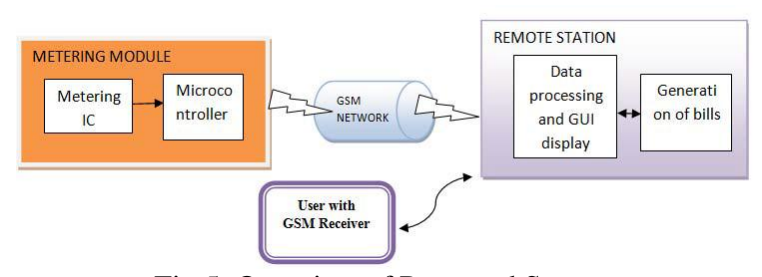

Fig.5. Overview of Proposed System.

The diagram gives the basic overview of automatic meter reading using GSM/GPRS [5] modem. The metering module consist of electronics meter and GSM modem embedded around microcontroller which is use for sending real time data to company through GSM network environment continuously. On the other hand, remote station contains database which stores the whole records coming from user side. On monthly basis bill get generated and send to the user by SMS, Email. One Web server also generated by which user can check his usage from any end of the world, for security purpose authentication provided to user. 


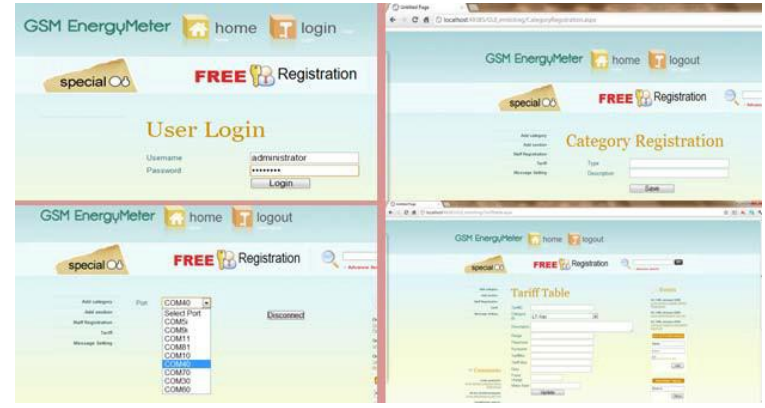

Fig.6. User login, Category registration, Port setting, Tariff table updating.

However the use of GSM in this particular system provides numerous advantages over methods that have been previously used. Data transmission is charged at standard SMS rates, thus the charges are not based on the duration of data transmission. The cost efficient transmission of readings ensures that power consumption values can be transmitted more frequently to a remote station. The implications of being able to transmit readings more often are that energy utilities will be able to generate timely bills, better understand energy demand patterns, manage meter failures more efficiently and manage fraud better. The developed system is highly effective in the sense it is able to eliminate the drawback of serial communication. i.e even though it lacks acknowledgement of the sent SMS it is not affecting system performance. If a message is missing then also as the system is accepting he cumulative value next time is which includes the lost content. And while preparing bill the system is accepting the maximum consumption value. These implementations make the designed system unique and effective compared to the previous proposals. Different state electricity boards in INDIA started using GSM facility for fault management and so there is increased demand for this method.

\section{CONCLUSIONS}

Automatic meter reading system has experienced significant changes in the past years. Different state electricity boards in INDIA like the Kerala State Electricity Board (KSEB) adopted this system for its fast, efficient and error free working. This paper describes the overviews of different technologies used for overcoming the problems and at last come up to this level. New technologies and methodologies which are already used to improve applications of AMR have been discussed in this paper. Till now PLC, Smart meters, WSN, GSM/GPRS are used in Automatic meter reading system.

\section{Possible Research Direction}

By using the Microchip three phase IC MCP3909 the same idea can be extended to three phase systems also. Power factor improvement options can be added in future. By adding an initialization message option at the time of installation the meter time can be updated from the server. If energy provider is willing to add online payment options in the hosted web page, instant payment by the consumer from anywhere in the world is also possible. The same idea can be expanded to water and gas meter reading system by proper modification. The designed web portal options can be added to the existing web page of any energy provider worldwide and it can be hosted for public.

\section{REFERENCES}

[1] Q. Gao et al., "Solutions for the "Silent Node" Problem in an Automatic Meter Reading System Using Power-Line Communications", IEEE vol. 23, no. 1, January 2008

[2] Subhashis Maitra et al., "Embedded Energy Meter- A New Concept To Measure The Energy Consumed By A Consumer And To Pay The Bill", 978-1-4244-1762-9/08 IEEE 2008

[3] Nhat-Quang Nhan et al., "Improving the performance of mobile data collecting systems for electricity meter reading using wireless sensor network", The 2012 International Conference on Advanced Technologies for Communications (ATC 2012) , pp. 241-246 [4] K. S. K. Weranga et al., "Smart Metering for Next Generation Energy Efficiency \& Conservation", IEEE PES ISGT Asia 2012, pp. 1-8 [5] Sudhish N George et al., "GSM Based Automatic Energy Meter Reading System with Instant Billing”, 978-1-4673-5090-7/13 IEEE 2013, pp. 61-72

[6] Mpendulo Ndlovu et al., "An OFDM Inter-Subcarrier Permutation Coding Scheme for Power-line Communication", 18th IEEE International Symposium on Power Line Communications and Its Applications, pp. 196-201, 2014

[7] Mohamed Chaker Bali et al., "Advanced Physical Layer for Robust Power Line Communications based on Coded Modulation", 9781-4799-5874-0/14, IEEE 2014

\section{BIOGRAPHIES}

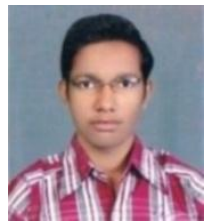

Bhushan D. Sawarkar is a M.E. Student of Priyadarshini College of Engineering, Nagpur (MH). He is completed his Bachelor of Engineering in Shree H.V.P.M.'s College of Engineering, Amravati. He got aggregate with $68 \%$ in whole year of B.E. Now Study of Master Degree from This College in Branch of Wireless Communication and Computing. And this paper all survey with Mrs. Snehal S. Golait.

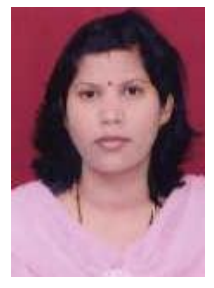

Mrs. Snehal S. Golait received her BE degree from Chandrapur College of Engineering and MTech degrees from G.H. Raisoni College of Engineering ,Nagpur . Currently she is pursuing her $\mathrm{PhD}$ in the Computer Science and Engineering from Nagpur University, Nagpur. Presently she is working as an Assistant Professor in Department of Computer Technology in Priyadarshini College of Engineering, Nagpur. She has published two papers in National Conference and 11 papers in International Conferences and 5 papers in International Journal. She has received "Excellance Award " in National Conference. Her research interests include Image Processing, Pattern recognition, Signal Processing. She has a teaching experience of 17 years. 